\title{
Coulomb implosion of tetrabromothiophene observed under multiphoton ionization by free-electron-laser soft-x-ray pulses
}

\author{
E. Kukk, ${ }^{1,2}$ H. Myllynen, ${ }^{1}$ K. Nagaya, ${ }^{3}$ S. Wada,${ }^{4}$ J. D. Bozek ${ }^{5}$ T. Takanashi, ${ }^{2}$ D. You, ${ }^{2}$ A. Niozu, ${ }^{3}$ K. Kooser, ${ }^{1}$ T. Gaumnitz, ${ }^{6}$ \\ E. Pelimanni, ${ }^{7}$ M. Berholts, ${ }^{1}$ S. Granroth, ${ }^{1}$ N. Yokono, ${ }^{3}$ H. Fukuzawa, ${ }^{2,8}$ C. Miron, ${ }^{9}$ and K. Ueda ${ }^{2,8}$ \\ ${ }^{1}$ Department of Physics and Astronomy, University of Turku, 20014 Turku, Finland \\ ${ }^{2}$ Institute of Multidisciplinary Research for Advanced Materials, Tohoku University, Sendai 980-8577, Japan \\ ${ }^{3}$ Department of Physics, Kyoto University, Kyoto 606-8502, Japan \\ ${ }^{4}$ Department of Physical Science, Hiroshima University, Higashi-Hiroshima 739-8526, Japan \\ ${ }^{5}$ Synchrotron SOLEIL, L'Orme des Merisiers, Saint-Aubin, BP 48, 91192 Gif-sur-Yvette Cedex, France \\ ${ }^{6}$ Laboratorium für Physikalische Chemie, ETH Zürich, 8093 Zürich, Switzerland \\ ${ }^{7}$ Nano and Molecular Systems Research Unit, Faculty of Science, 90014 University of Oulu, Finland \\ ${ }^{8}$ RIKEN SPring-8 Center, Kouto 1-1-1, Sayo, Hyogo 679-5148, Japan \\ ${ }^{9}$ LIDYL, CEA, CNRS, Université Paris-Saclay, CEA Saclay, 91191 Gif-sur-Yvette, France
}

(Received 1 October 2018; published 11 February 2019)

\begin{abstract}
Soft-x-ray free-electron-laser pulses were used to create highly charged molecular tetrabromothiophene species by sequential multiphoton ionization from bromine $3 d$ orbitals. The experiment was performed at the SACLA facility in Japan and the products of molecular dissociation were analyzed by means of multicoincidence momentum-resolved ion time-of-flight spectroscopy. Total charge states up to +13 atomic units were produced, creating a particular dissociation pattern for the carbon ions, a Coulomb implosion, due to the concerted forces by the surrounding heavy bromine ions. This behavior was explored both experimentally and by numerical molecular-dynamics simulations and the fingerprints of the Coulomb implosion were identified in both. In simulations, Coulomb implosion was predicted to be highly sensitive to the initial (thermal) motion of the atoms and, after including vibrational motion for several temperatures, good general agreement between the experiment and simulations was found. The agreement with the experiment was further improved by adding charge dynamics to the simulation, according to our point-charge dynamics model with empirical rate constants.
\end{abstract}

DOI: 10.1103/PhysRevA.99.023411

\section{INTRODUCTION}

Since the very first spectroscopy experiments using x-ray free-electron lasers (FELs) [1-5], the femto- and picosecond dynamics of the Coulomb explosion of small quantum systems from diatomic molecules to rare-gas clusters has captured continuous interest [6-13]. Ionization by the short and intense FEL pulses allows creating exotic highly charged states by depositing a large amount of energy into the system in the course of tens of femtoseconds or even less. The ensuing dynamics is a complex process, encompassing charge multiplication by electronic decay, charge redistribution within the system, and nuclear dynamics, all overlapping in time. Various theoretical descriptions and models can be applied in the interpretation of such experiments [13-15], ranging from $a b$ initio quantum chemical molecular-dynamics simulations to simple classical point-charge models.

In our earlier works, we investigated molecules with one or two x-ray-absorption hot spots, i.e., iodine atoms in iodomethane [16], di-iodomethane [17], and iodouracil $[18,19]$, and applied a Coulomb explosion model with a few empirical parameters representing the charge dynamics. The experimental technique used was multi-ion coincidence timeof-flight spectroscopy with three-dimensional momentum resolution [20-22]. The empirical Coulomb explosion model was in good agreement with the various experimental findings related to the dissociation dynamics, such as ion momentum distributions and angular correlations between the momenta of various fragments. In these studies, the focus was on the interplay of the charge and nuclear dynamics, for example, on how the dissociation pattern is affected by the continuous buildup and spread of the positive charge, as opposed to the instant charge appearance over the entire molecule. This aspect of joint charge and nuclear dynamics is especially important when the nuclear dynamics has an ultrafast component (proton emission) and the charge is multiplied by multistep Auger cascades from deep core holes in the absorption hot spots, such as I $2 p$ core holes.

In smaller systems such as $\mathrm{CH}_{3} \mathrm{I}$, the initial vibrational motion of the constituent atoms has only a very minor impact on the Coulomb explosion, quite understandably when one compares the typical total internal vibrational energy (less than $200 \mathrm{meV}$ in $\mathrm{CH}_{3} \mathrm{I}$ ) with the energy released in the Coulomb explosion (about $250 \mathrm{eV}$ in $\mathrm{CH}_{3} \mathrm{I}^{10+}$ ). However, in a more complex system of 5-iodouracil, it was found that in order to interpret the experimentally determined momentum and angular distributions of the fragments correctly, it is necessary to also include the initial motion of the atoms $[18,19]$. In the present study, we will further investigate the role of the molecular vibrations in a system with rather unique fragmentation dynamics. 


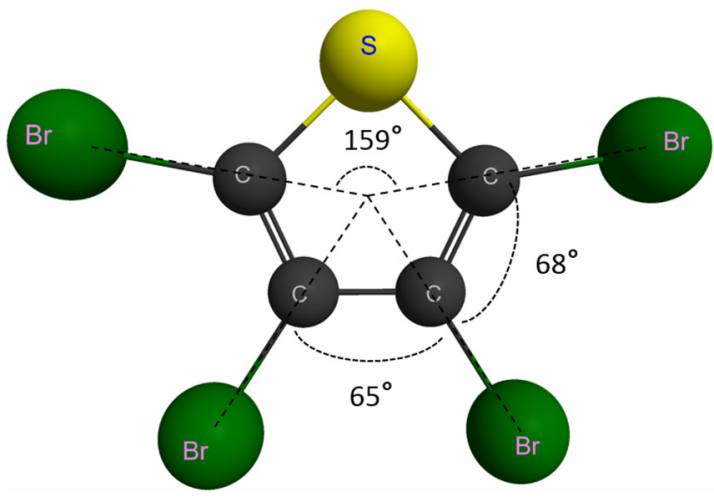

FIG. 1. Geometry of the 4BrThio molecule.

The target molecule in this study is tetrabromothiophene (4BrThio), where all the hydrogens surrounding the thiophene ring have been replaced with $\mathrm{Br}$ atoms (see Fig. 1). This means that there is no ultrafast nuclear dynamics component. Another contrast with the previous studies at SACLA is that the molecule was ionized by soft-X-ray FEL pulses that at $130 \mathrm{eV}$ predominantly create the shallow $\mathrm{Br} 3 d$ core holes that decay rapidly by single-step molecular Auger processes. Combined with the lack of ultrafast nuclear dynamics, one can assume that in the present system, the faster charge dynamics has a relatively minor effect on the dissociation dynamics.

By tuning the pulse intensity to the multiphoton absorption regime, a highly symmetric situation can be created where the carbons in the thiophene ring are surrounded by heavy $\mathrm{Br}^{+}$ ions. What follows is, according to the simulation, a picture where the bromine ions follow the usual Coulomb explosion trajectories reflecting the molecular geometry, but the carbon ions experience a Coulomb implosion. They become trapped in the cage of heavy bromine atoms with the Coulomb forces acting inward. In such a situation even a small initial displacement or velocity of carbon atoms can dramatically alter the ion emission trajectories.

In the following, we will first present the experimental findings as a basis to reconstruct the dissociation behavior with the aid of modeling. Then we calculate the ion momenta by classical point-charge simulation in order to determine the typical total molecular charge reached in the experiment. Next the initial vibrational motion will be added to the model and compared with the experiment using the most symmetric charge situation with each atom singly charged, which is also representative of the experimental conditions. Last, we will add charge dynamics to the simulations.

\section{EXPERIMENT}

\section{A. Experimental setup and conditions}

The experiments were carried out at beamline 1 (BL1) [23] of the SACLA free-electon-laser facility [24] operating at a repetition rate of $60 \mathrm{~Hz}$. The pulse duration of the x-ray FEL was estimated to be about 70-fs full width at half-maximum. The purpose of the experiment was to rapidly create a highly charged state of 4BrThio, especially by charging all four bromine atoms. Therefore, the photon energy of $130 \mathrm{eV}$ was chosen near the maximum cross section for $\mathrm{Br} 3 d$ ionization while still well within the tuning range of the BL1 of SACLA. At $130 \mathrm{eV}$, the total photoabsorption cross section for 4BrThio is $24 \mathrm{Mbarns}$ (estimated from the atomic cross sections [25]) and $88 \%$ of it is $\mathrm{Br} 3 d$ ionization.

The average nonattenuated pulse energy during the experiment was $50 \mu \mathrm{J}$, corresponding to $2.4 \times 10^{12}$ photons per FEL pulse, focused a Kirkpatrick-Baez mirror system through a waist of $5.3 \times 5.1 \mu \mathrm{m}^{2}(v \times h)$ diameter $(\mathrm{FWHM})$. The described conditions would correspond to a very high multiphoton absorption regime, where the total molecular charge created per pulse becomes limited by the effect of frustrated absorption. In order to limit the ionization of the residual gas, and also because the very high photon density is not necessary for sufficient ionization of the sample, the photon flux was limited by metal foil filters. The main measurement was performed using a $\mathrm{Sn} 0.1-\mu \mathrm{m}$ filter with calculated $24 \%$ transmission and a complementary measurement using a $0.2-\mu \mathrm{m}$ filter with calculated $5.4 \%$ transmission at the chosen energy. The transmissions as determined using gas pulse energy monitors were higher, $71 \%$ and $30 \%$, correspondingly.

4BrThio (greater than $99.0 \%$ purity) was purchased from Tokyo Chemical Industry Co., Ltd., and used without further purification. The solid sample was heated up to $120^{\circ} \mathrm{C}$ in the reservoir of a modified electromagnetic solenoid valve [26]. The resulting sample vapor was seeded by helium gas and introduced to the focal point of the FEL pulses in the ultrahigh-vacuum reaction chamber as a pulsed supersonic gas jet. The ions were detected by a multicoincidence momentumimaging ion time-of-flight (TOF) spectrometer equipped with a Roentdek HEX120 position-sensitive ion detector [21]. The acceleration voltages were tuned to velocity mapping conditions with the $4 \pi$ ion collection efficiency up to $35 \mathrm{eV}$ initial kinetic energy.

\section{B. Results \\ 1. Ionic fragments}

As the first step of the analysis, the fragment ions originating from the $4 \mathrm{BrThio}$ sample were identified. This was not a straightforward process, since most of the signal in the ion TOF spectra came from the residual gas. However, the signal from the $\mathrm{Br}^{+}$ions is well separated from any rest-gas peaks and, in the dissociation of multiply charged 4BrThio, most events are expected to yield $\mathrm{Br}^{+}$ions. Since only $20 \%$ of the FEL pulses yielded any $\mathrm{Br}^{+}$ions, it is likely that for the rest there were no 4BrThio molecules in the interaction volume at the time of the pulse. In order to obtain a TOF spectrum assigned only to the 4BrThio sample, the $\mathrm{Br}^{+}$ions were used as a filter, generating an ion TOF spectrum only from events containing one or more $\mathrm{Br}^{+}$ions and then subtracting a TOF spectrum generated from events containing no $\mathrm{Br}^{+}$ions. The result given in Fig. 2 shows which ionic species correlate with the $\mathrm{Br}^{+}$signal. The figure also displays the original TOF spectrum generated without filtering. The spectral structures are labeled by the assigned ionic species. $\mathrm{The}^{+} \mathrm{Br}^{+}$peak is composed of two isotopes, ${ }^{79} \mathrm{Br}$ and ${ }^{81} \mathrm{Br}$, indicated by two markers. As expected, also the other atomic fragments $\mathrm{C}^{+}$ and $\mathrm{S}^{+}$are present, as well as the $\mathrm{Br}^{2+}$ ions. Also the $\mathrm{C}_{2}{ }^{+}$ fragment is seen in significant quantity and a small amount of $\mathrm{CBr}^{+}$fragments. The disturbed region following the $\mathrm{C}^{+}$peak 


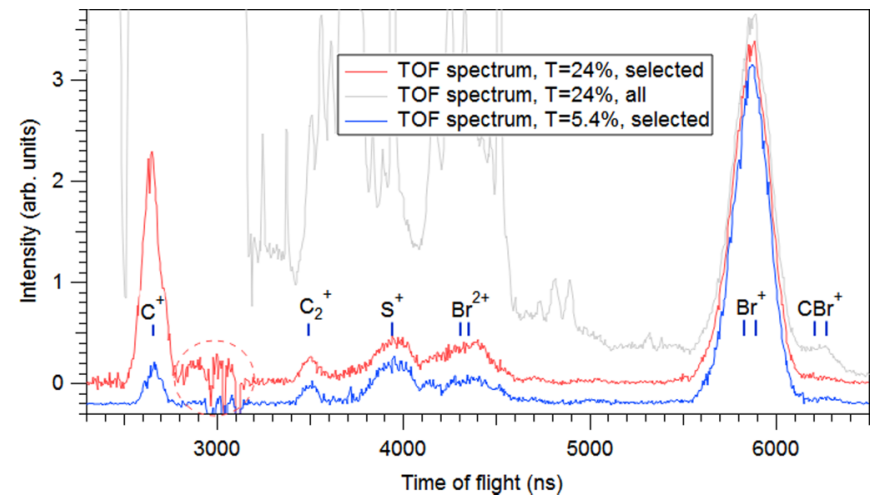

FIG. 2. Ion TOF spectrum of 4BrThio. Colored lines are spectra generated from selected events producing at least one $\mathrm{Br}^{+}$ion and the light gray line is the spectrum generated from all events. The red (upper) spectrum shows a spectrum with the FEL pulses attenuated to $24 \%$ and the blue (lower) spectrum (offset for better visibility) to $5.4 \%$ of the full intensity. The TOF positions of various fragments, corresponding to zero initial velocity, are marked by vertical lines. The dashed circle denotes the region heavily disturbed by the water molecular ion and fragments.

is due to the subtraction of a very strong signal from water ions. The peak assigned to ${ }^{79,81} \mathrm{Br}^{+}$can in principle be also assigned to the $\mathrm{C}_{4} \mathrm{~S}^{+}$fragment, but it is very unlikely that the thiophene ring survives intact. The fragment intensity ratios in the figure are not representative of the true ion abundances, as the filtering process creates a bias towards $\mathrm{Br}$ ions.

\section{Momentum distributions}

As can be seen from Fig. 2, all the TOF peaks are significantly broadened, an indication of a large momentum component of the ions along the spectrometer's axis. Also, the ion hit position distributions on the position-sensitive detector are broad due to the transverse momentum component, e.g., the image of $\mathrm{Br}^{+}$ions has a two-dimensional Gaussian width of about $18 \mathrm{~mm}$.

Numerical ion flight trace simulations were used to obtain accurate conversion coefficients from TOF deviations $\triangle \mathrm{TOF}$, $x$ and $y$, to all three components of $\vec{p}$. Figure 3 shows the momentum distributions of the $\mathrm{Br}^{+}$and $\mathrm{C}^{+}$ions. Unfortunately, due to strong contamination from residual gas, the distributions for other ions could not be reliably extracted. For the $\mathrm{Br}^{+}$ions, the presence of two unresolved isotopes introduces uncertainty in determining the axial component of the ion momentum from $\triangle \mathrm{TOF}$, whereas the other two components are virtually unaffected by the isotopic distribution. A numerical simulation shows that using the average mass of 80 atomic mass units (u) in analysis results in an additional broadening of the momentum distribution, which is about $3 \times 10^{-23} \mathrm{~kg} \mathrm{~m} / \mathrm{s}$ in the higher-momentum side and $(1-2) \times 10^{-23} \mathrm{~kg} \mathrm{~m} / \mathrm{s}$ in the lower-momentum side of the distribution. The isotope effect thus does not have a significant influence on the analysis.

The solid lines in Fig. 3 are the momentum distributions for all detected $\mathrm{Br}^{+}$or $\mathrm{C}^{+}$ions, but it is informative to study also filtered subsets. The red dashed lines in Fig. 3 correspond to the $\mathrm{Br}^{+}$ions coming only from the FEL pulses with at

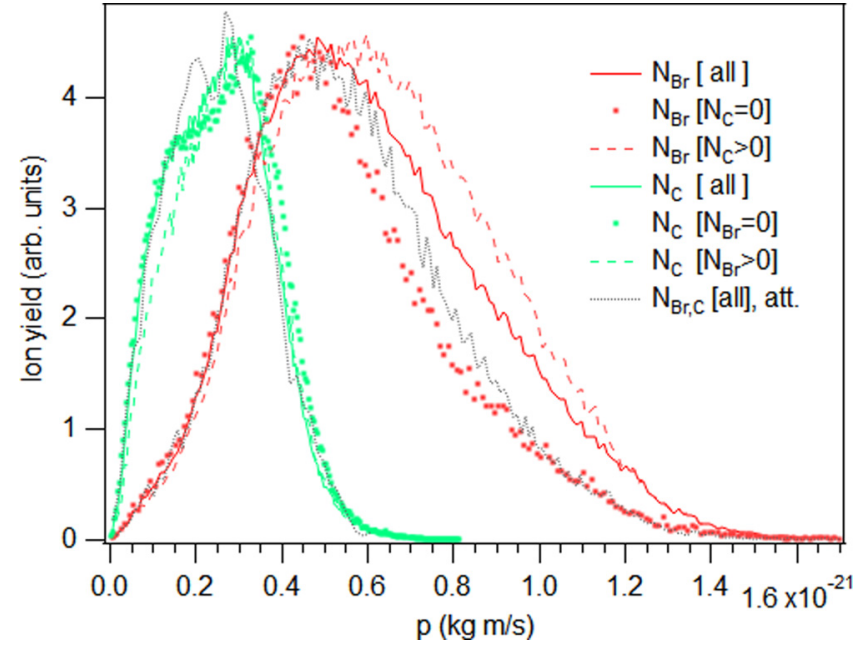

FIG. 3. Ion momentum distributions for $\mathrm{Br}^{+}$(red) and $\mathrm{C}^{+}$ (green) from the experiment with $24 \%$ pulse attenuation. Solid lines are distributions for the ions from all FEL pulses, dashed lines for the $\mathrm{Br}^{+}$and $\mathrm{C}^{+}$ions that have counterpart $\mathrm{C}^{+}$and $\mathrm{Br}^{+}$ions, respectively, in the FEL pulse, and dotted lines for the $\mathrm{Br}^{+}$and $\mathrm{C}^{+}$ions that have no counterpart ion in the pulse. The gray dotted lines show momentum distributions from the experiment with stronger $5.4 \%$ attenuation.

least one $\mathrm{C}^{+}$ion present and the dotted lines correspond to the $\mathrm{Br}^{+}$ions from pulses with no $\mathrm{C}^{+}$ions present. A comparison of the two subsets shows that the existence of $\mathrm{C}^{+}$ions in a pulse correlates with the $\mathrm{Br}^{+}$ions with larger momentum. The equivalent analysis for the $\mathrm{C}^{+}$momentum distribution has quite a different outcome: There are slightly more energetic ions in the $\mathrm{C}^{+}$distribution when there are no $\mathrm{Br}^{+}$ions present, compared to the unfiltered case or the case where there is at least one $\mathrm{Br}^{+}$in the pulse. Such behavior of both distributions can be explained if, by dividing the total charge between the atomic fragments, $\mathrm{Br}$ atoms are initially preferred over the $\mathrm{C}$ atoms. Then the presence of $\mathrm{C}^{+}$ions together with the $\mathrm{Br}^{+}$ion indicates that relatively high total charge states are reached and the average momenta of the $\mathrm{Br}^{+}$ions are higher, while the presence of $\mathrm{Br}^{+}$ions together with $C^{+}$ions would not be a clear indication of either higher or lower total charge. Also, the correlation with the total charge state is not so clear-cut for the momentum of the $\mathrm{C}^{+}$ions, as our simulations will show (see Fig. 6).

Figure 3 shows also the momentum distributions obtained by the more strongly attenuated FEL pulses. For $\mathrm{Br}^{+}$, this clearly reduces the high-momentum fraction of the ions, consistent with lower total charge being produced. For $\mathrm{C}^{+}$, similarly to filtering of events by $\mathrm{Br}^{+}$ions, the effect is more in the detailed shape of the distribution.

\section{Momentum correlations}

The angular correlations between the momentum vectors in $\left(\mathrm{Br}^{+}, \mathrm{Br}^{+}\right)$and $\left(\mathrm{C}^{+}, \mathrm{C}^{+}\right)$ion pairs are shown in Fig. 4, with $\alpha$ the angle between the two vectors. Here all possible ion pairs belonging to the same FEL pulse are included; therefore the curves are expected to have a contribution also from false coincidence pairs. As a reference and cross-check, distributions were also created from scrambled ion pairs, 


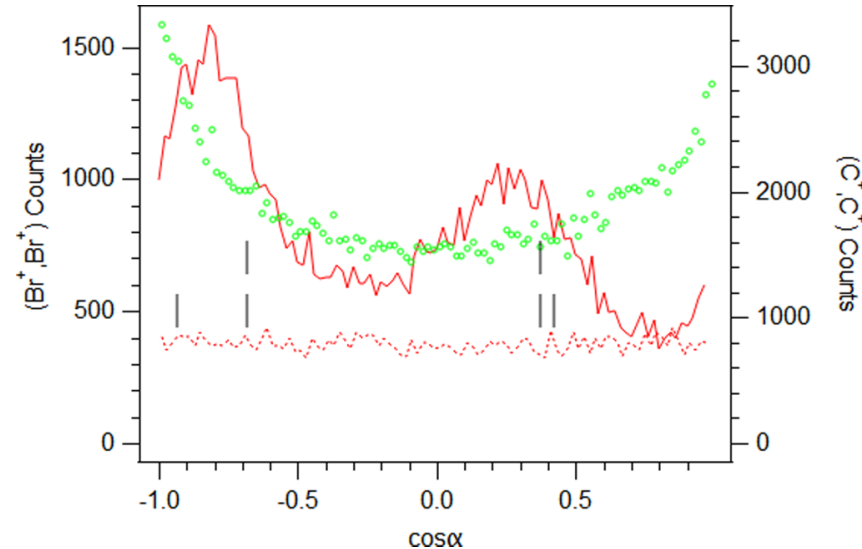

FIG. 4. Distribution of the cosine of the angle between the momentum vectors of ions created by a FEL pulse. The solid red line shows $\left(\mathrm{Br}^{+}, \mathrm{Br}^{+}\right)$pairs and green circles $\left(\mathrm{C}^{+}, \mathrm{C}^{+}\right)$pairs. The dotted line shows the distributions from scrambled $\left(\mathrm{Br}^{+}, \mathrm{Br}^{+}\right)$(red) and $\left(\mathrm{C}^{+}, \mathrm{C}^{+}\right)$(green) pairs. Vertical markers indicate the $\mathrm{C}-\mathrm{Br}$ bond angles from the neutral geometry.

where the ions were picked from different FEL pulses, therefore eliminating any possibility of true coincidences. As expected, these distributions are entirely flat in the $\cos \alpha$ presentation, while the true $\left(\mathrm{Br}^{+}, \mathrm{Br}^{+}\right)$pairs show two clear maxima. The black markers indicate the cosines of the angles between all the $\mathrm{C}-\mathrm{Br}$ bonds in the neutral ground-state geometry of the molecule. The experimental maxima of the $\left(\mathrm{Br}^{+}, \mathrm{Br}^{+}\right)$pairs indeed correlate with these angles, while the angular distribution of the $\left(\mathrm{C}^{+}, \mathrm{C}^{+}\right)$pairs shows a very different shape, with no correlation to the ground-state geometrical positions of the $\mathrm{C}$ atoms in the thiophene ring. Instead, the momenta of the $\mathrm{C}^{+}$ions tend to be preferentially close to parallel or antiparallel to each other.

Let us now consider the effects of the planar symmetry of the 4BrThio molecule. If all atoms lie strictly on a plane at the start of dissociation, with no initial velocities, then the entire dissociation dynamics would take place on that molecular plane. Experimentally, three-ion coincidences (ion triplets) would provide a means to check the planarity of the dynamics, with the momentum vectors of the first two ions defining a plane and the measure of planarity defined as the cosine of the angle $\beta$ between the normal of the plane and the momentum of the third ion. A distribution sharply peaked at $\cos \beta=0$ would mean all three ions dissociating preferentially on a plane.

Figure 5 shows the $\cos \beta$ distributions from the triplets $\left(\mathrm{Br}^{+}, \mathrm{Br}^{+}, \mathrm{Br}^{+}\right)$and $\left(\mathrm{Br}^{+}, \mathrm{Br}^{+}, \mathrm{C}^{+}\right)$, again showing a flat scrambled distribution of $\left(\mathrm{Br}^{+}, \mathrm{Br}^{+}, \mathrm{Br}^{+}\right)$and $\left(\mathrm{Br}^{+}, \mathrm{Br}^{+}, \mathrm{C}^{+}\right)$as a cross-check. The distributions contain an uncorrelated (flat) false coincidence background.

One can see that the three $\mathrm{Br}^{+}$ions indeed dissociate preferentially on a plane, reaffirming that their dissociation characteristics reflect the original geometry of the molecule. The deviations from the planar dissociation vary by $\pm 22^{\circ}$ (half-width at half maximum of the distribution). For the $\mathrm{C}^{+}$ions, in contrast, the preferred momentum direction is perpendicular to the plane defined by the two $\mathrm{Br}^{+}$momentum vectors, symmetrically above $(\cos \beta=1)$ or below $(\cos \beta=$

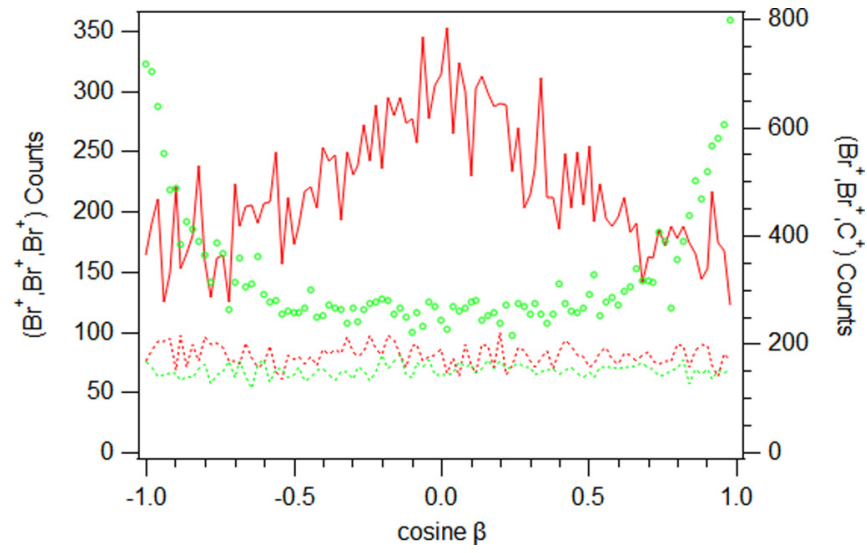

FIG. 5. Distribution of the cosine of the angle between the normal of the plane defined by the momenta of two ions and the momentum of a third ion. The solid red line shows $\left(\mathrm{Br}^{+}, \mathrm{Br}^{+}, \mathrm{Br}^{+}\right)$ triplets, green circles show $\left(\mathrm{Br}^{+}, \mathrm{Br}^{+}, \mathrm{C}^{+}\right)$triplets, and dotted red and green lines show scrambled $\left(\mathrm{Br}^{+}, \mathrm{Br}^{+}, \mathrm{Br}^{+}\right)$and $\left(\mathrm{Br}^{+}, \mathrm{Br}^{+}, \mathrm{C}^{+}\right)$ triplets, respectively.

$-1)$. This is also in agreement with the two-ion angular correlations for carbon fragments (Fig. 4); as the $\mathrm{C}^{+}$ions leave the plane of the molecule, all angular relationship with the initial geometry on the plane is lost.

\section{MOLECULAR-DYNAMICS MODELING}

\section{A. Charge distributions}

Our Coulomb explosion model is a fairly simple one that has earlier been successfully used to model the momentum distribution of iodomethane [16], di-iodomethane [17], and 5iodouracil [19] upon ionization. In the model, the total charge of the molecule is localized at single atoms which are assumed to behave like point charges without any chemical bonds. In the first approximation, the total charge is distributed to atoms immediately without any charge buildup time. In a more advanced form, the charge builds up gradually. For simplicity, we will first apply the model with instantaneous charge creation, a fair approximation due to the lack of ultrafast dissociation component.

From the experiment it is not apparent how large a total charge is reached in the ionization, so first we obtained the momenta for different total charges using the instantaneous charge creation approximation. An average mass $80 \mathrm{u}$ was used for the $\mathrm{Br}$ atoms and the equations of motion were integrated up to $100 \mathrm{ps}$. The $\mathrm{C}^{+}$and $\mathrm{Br}^{+}$momenta are plotted in Fig. 6 for total charge values up to $Q_{\text {tot }}=13$, overlaid on the experimental momentum distributions of these ions. The spread of the possible momentum values in simulation at each $Q_{\text {tot }}$ comes from the permutations of the charge distribution between individual atoms. In Fig. 6, two scenarios are presented. In Fig. 6(a), the Br atoms are preferred for charge localization, so the $\mathrm{C}$ atoms start to obtain charge only after all four $\mathrm{Br}$ atoms have +1 charge. In Fig. 6(b), the charge can always localize to all atoms with equal probability, with the constraint that doubly charged atoms are generated only after all atoms have become singly ionized. In both cases, all atoms obtain the unit charge at a total charge of +9 , with no permutations possible, and the highest atomic charge is +2 . 

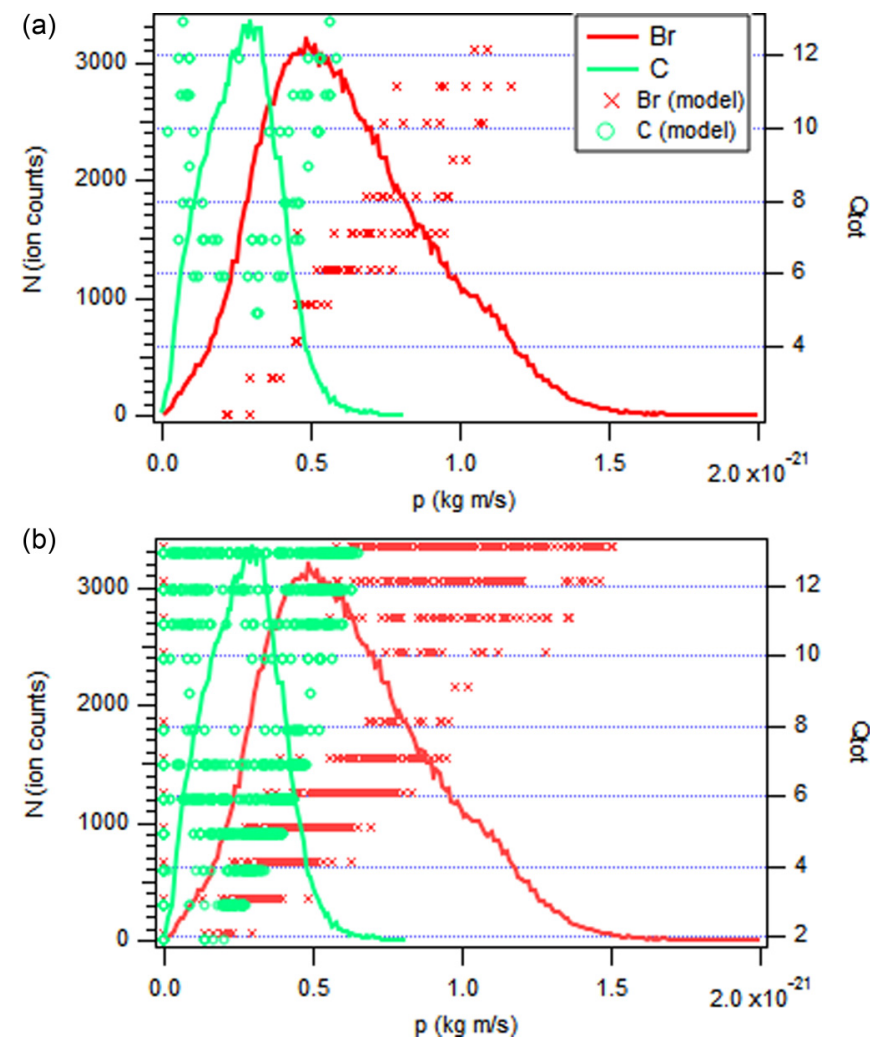

FIG. 6. Momentum distributions of $\mathrm{Br}^{+}$and $\mathrm{C}^{+}$ions from 4BrThio in the experiment and from simulations with the total charge $Q_{\text {tot }}$ from +2 to +13 . Solid lines are the experimental curves and markers show the momenta given by the model. The markers in (a) are the possible momenta when the charge on $\mathrm{Br}$ atoms is preferred over other atoms, whereas in (b) all atoms have equal likelihood to be charged.

Additionally, different ion momentum values arise since there are two inequivalent positions for $\mathrm{Br}$ and $\mathrm{C}$ atoms in the geometry.

The simulated momenta give the first indication of the total charge states covered by the present experiments. The maximum in the experimental distribution of $\mathrm{Br}^{+}$corresponds to modeled values for $Q_{\text {tot }}=5, \ldots, 6$, with the distribution extending to cover the total charge $Q_{\text {tot }}=13$. The used instantaneous charge approximation gives the upper limits of the momenta and adding a charge buildup time will lower these values, as will be seen in Sec. III C. From the experiment (Fig. 3), it was shown that the larger values in the $\mathrm{Br}^{+}$ momenta correspond to the more uniform charge distribution with the $\mathrm{C}^{+}$counterpart also present. All these considerations suggest that, when selecting a single total charge value as representative of the experiment in further simulations, $Q_{\mathrm{tot}}=$ 9 would be not only suitable but also very advantageous since (i) there is no ambiguity in distributing +1 charges and (ii) it creates a most symmetrical situation in terms of the Coulomb forces and the perfect conditions for the Coulomb implosion. In the following modeling, we used $Q_{\mathrm{tot}}=9$.

\section{B. Angular correlations: Effects of temperature}

Even though the sample temperature was measured during the experiment, the exact internal temperature of the 4BrThio
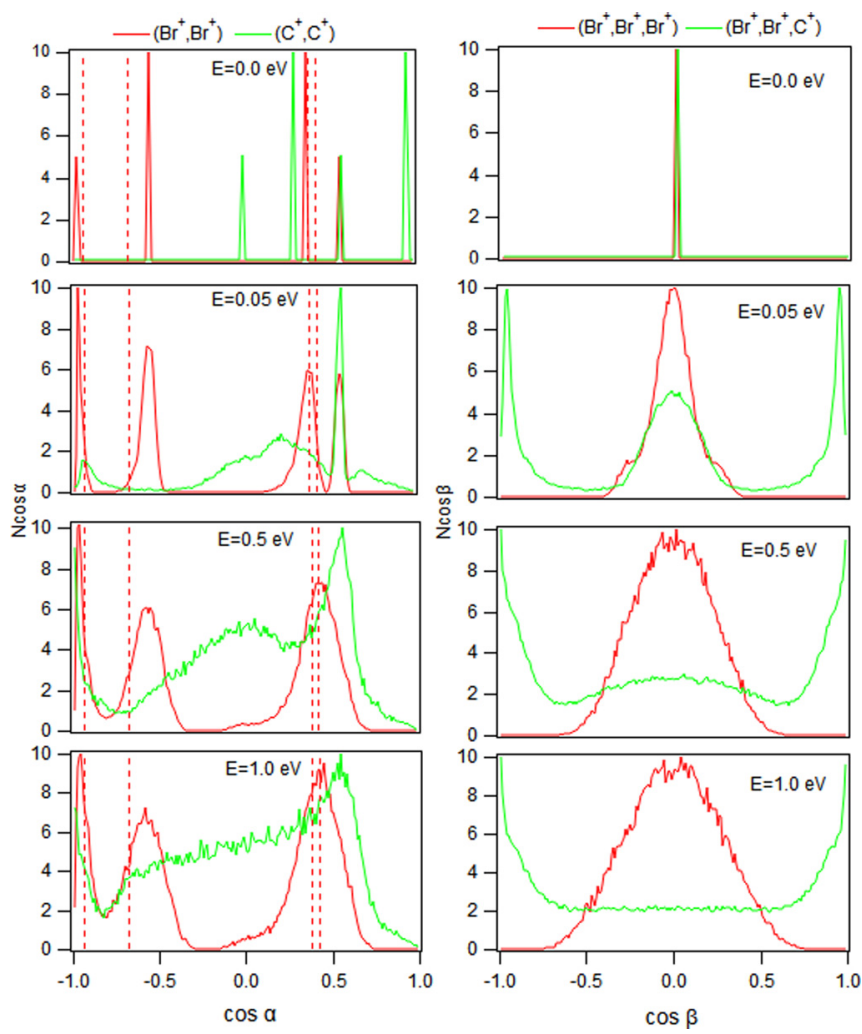

FIG. 7. Angle distributions of $\left(\mathrm{Br}^{+}, \mathrm{Br}^{+}\right)$and $\left(\mathrm{C}^{+}, \mathrm{C}^{+}\right)$pairs $(\cos \alpha)$ and the triplets $\left(\mathrm{Br}^{+}, \mathrm{Br}^{+}, \mathrm{Br}^{+}\right)$and $\left(\mathrm{Br}^{+}, \mathrm{Br}^{+}, \mathrm{C}^{+}\right)(\cos \beta)$. The initial thermal energy is increased from $0 \mathrm{eV}$ to $1 \mathrm{eV}$ from top to bottom. Vertical dashed lines indicate the cosines of the angles between the $\mathrm{C}-\mathrm{Br}$ bonds.

molecules in the expanding jet was not known. The sample evaporation temperature was around $400 \mathrm{~K}$, which corresponds to about $0.5 \mathrm{eV}$ total kinetic energy for the atoms in the 4BrThio molecule, calculated from the equipartition theorem as $E_{\mathrm{kin}}=N \times \frac{3}{2} k_{B} T$, where $N$ is the number of atoms in the molecule, $k_{B}$ is Boltzmann constant, and $T$ is the temperature. This energy was added to all atoms as randomized, isotropically oriented initial velocity vectors, so the total atomic kinetic energy adds up to the predetermined value (e.g., $0.5 \mathrm{eV}$ for $400 \mathrm{~K}$ ) and the center of mass remains stationary. In the simulations, 10000 trajectories were integrated for each temperature, varying the ensemble of the initial velocity vectors.

Next we compare simulations at a few different thermal energies. The angular distributions of the $\left(\mathrm{Br}^{+}, \mathrm{Br}^{+}\right)$ and $\left(\mathrm{C}^{+}, \mathrm{C}^{+}\right)$pairs and of the $\left(\mathrm{Br}^{+}, \mathrm{Br}^{+}, \mathrm{Br}^{+}\right)$and $\left(\mathrm{Br}^{+}, \mathrm{Br}^{+}, \mathrm{C}^{+}\right)$triplets are shown in Fig. 7 in the left and right columns, respectively. With no initial velocities $(T=$ $0 \mathrm{~K}$ ), and since all Coulomb forces are on the molecular plane, all departing ions stay on that plane. As the top row of Fig. 7 shows, ions in both the $\mathrm{Br}^{+}$and $\mathrm{C}^{+}$ion pairs leave at angles that are defined by the initial geometry and the charge distribution (which in this case is uniformly +1 per atom). Typical Coulomb explosion often reflects the bond angles in the initial geometry (bond breakage), therefore also in Fig. 7 the four possible (cosines of the) angles between the $\mathrm{C}-\mathrm{Br}$ bonds are indicated. As can be seen, although the 
geometry fully determines the angles in this simulation, the correspondence to the original geometry in the $\left(\mathrm{Br}^{+}, \mathrm{Br}^{+}\right)$ case is only approximate and for the $\left(\mathrm{C}^{+}, \mathrm{C}^{+}\right)$pairs it is not present at all. It is interesting to note that there are $\left(\mathrm{C}^{+}, \mathrm{C}^{+}\right)$ pairs with the momenta almost codirectional $(\cos \alpha=0.95)$. These are formed by the lower and upper (attached to sulfur) carbons (Fig. 1). With no vibrational motion, the former undergo multiple scattering inside the $\mathrm{Br}^{+}$and $\mathrm{S}^{+}$cage and eventually leave with very low kinetic energy of less than $1 \mathrm{eV}$. This reduces the Coulomb repulsion with the faster carbon ions and allows for nearly codirectional emission, as a peculiarity of this particular geometry and the chosen charge distribution.

When even a small amount of vibrational energy is added to the initial state, the behavior is changed as shown in the second row of Fig. 7, showing the case of $40 \mathrm{~K}$ temperature ( $0.05 \mathrm{eV}$ internal energy). In the left-hand figure, we see that the $\mathrm{C}^{+}$ions no longer form the four sharp peaks that were present in the $0-\mathrm{eV}$ case, but the distribution of $\left(\mathrm{C}^{+}, \mathrm{C}^{+}\right)$now has a broad structure and a new maximum near at $\cos \alpha=-1$ appears. Only one of the four original peaks is still distinct at $\cos \alpha=0.57\left(\alpha=55^{\circ}\right)$; it is almost totally formed by the two equivalent carbons that were bonded to sulfur. In contrast, the four $\left(\mathrm{Br}^{+}, \mathrm{Br}^{+}\right)$peaks seen at $0 \mathrm{eV}$ energy are still present at $0.05 \mathrm{eV}$, though slightly broadened. Turning to the planarity of the dissociation, one sees from the right-hand column of row 2 in Fig. 7 that whereas in the zero-energy case the $\cos \beta$ angles of all triplets $\left(\mathrm{Br}^{+}, \mathrm{Br}^{+}, \mathrm{Br}^{+}\right)$and $\left(\mathrm{Br}^{+}, \mathrm{Br}^{+}, \mathrm{C}^{+}\right)$are strictly zero (planar emission), now other maxima appear in the $\left(\mathrm{Br}^{+}, \mathrm{Br}^{+}, \mathrm{C}^{+}\right)$triplets near $\cos \beta= \pm 1$, indicating that $\mathrm{C}^{+}$ions are emitted close to the normal of the molecular plane. These effects of temperature have a simple explanation: The heavy and thus slower $\mathrm{Br}^{+}$ions are blocking the Coulomb explosion path of the $\mathrm{C}^{+}$ion on the molecular plane. Even a small disturbance of the initial planar symmetry creates an off-plane force on the $\mathrm{C}^{+}$ions that soon becomes the dominant force component: The carbon ions will "pop out" of the molecular plane and also lose all directional correlation with the initial molecular geometry. However, at that low temperature of $40 \mathrm{~K}$, some $\mathrm{C}^{+}$ions still stay at the molecular plane causing the central maximum around $\cos \beta=0$. About $97 \%$ of that peak comes from the two C atoms that were bonded to the $\mathrm{S}$ atom and that also created the one remaining clear peak in the $\cos \alpha$ distribution. The side maxima at $\cos \beta= \pm 1$ are caused almost totally by the two other $\mathrm{C}$ atoms. The $\mathrm{Br}^{+}$ions, in contrast to carbon, are still emitted close to the molecular plane as their $\cos \beta$ distribution peaks around zero, although it is broadened by the initial thermal motion, having the variation from planar as $\pm 6^{\circ}$ half-width at half maximum (HWHM).

At the next temperature, $E=0.5 \mathrm{eV}$ corresponding to $400 \mathrm{~K}$ (the third row of Fig. 7), the peak for $\left(\mathrm{C}^{+}, \mathrm{C}^{+}\right)$at $\cos \alpha=0.57$ in the left-hand figure broadens further. A peak at $\cos \alpha=-1.0$ that is now well defined is created by the $\mathrm{C}^{+}$ ions emitted to the opposite sides of the molecular plane. In the right-hand column of Fig. 7, the in-plane maximum at $\cos \beta=0$ nearly disappears for $\left(\mathrm{Br}^{+}, \mathrm{Br}^{+}, \mathrm{C}^{+}\right)$and the $\mathrm{C}^{+}$ ions now mostly leave near perpendicular to the plane defined by the bromine momenta. All the $\mathrm{Br}^{+}$ions are still emitted preferentially in the planar configuration, although now the

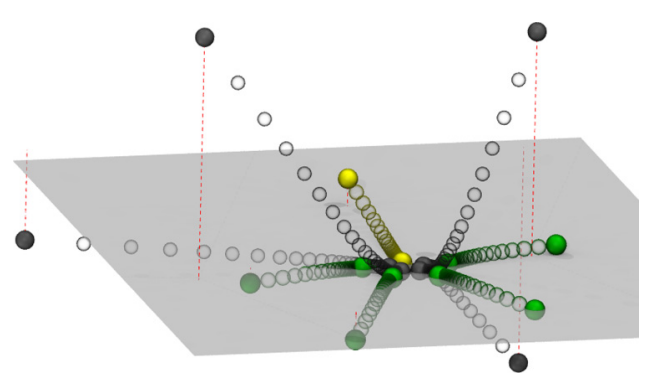

FIG. 8. Example of the fragmentation trajectory of 4BrThio with $Q_{\text {tot }}=9$ during the first $200 \mathrm{fs}$, with $0.5 \mathrm{eV}$ of initial thermal kinetic energy distributed to the atoms: $\mathrm{Br}$ atoms, green; $\mathrm{C}$ atoms, gray; and $\mathrm{S}$ atoms, yellow. Dashed red lines show the distance to the plane defined by the initial geometry of the molecule.

variation is $\pm 18^{\circ}$ from the plane (HWHM). In experiment, the observed variation was $\pm 22^{\circ}$.

The different behavior of the $\mathrm{C}^{+}$and $\mathrm{Br}^{+}$ions is illustrated by Fig. 8 , showing the simulated ion trajectories during the first $200 \mathrm{fs}$ of the dissociation of a uniformly charged, $Q_{\mathrm{tot}}=$ 9, 4BrThio molecule at one randomly chosen set of initial velocities corresponding to $400 \mathrm{~K}(0.5 \mathrm{eV})$. The $\mathrm{Br}^{+}$ions stay close the initial molecular plane with the trajectories reflecting the initial $\mathrm{C}-\mathrm{Br}$ bond directions. It is seen how three $\mathrm{C}^{+}$ions "bounce off" the heavy $\mathrm{Br}^{+}$ions and turn out of the plane, whereas one $\mathrm{C}^{+}$ion, initially bonded to $\mathrm{S}$, leaves close to the molecular plane, illustrating the entire range of the $\left(\mathrm{Br}^{+}, \mathrm{Br}^{+}, \mathrm{C}^{+}\right)$curve in the third row of Fig. 7 .

The bottom row of Fig. 7 represents the highest temperature of $800 \mathrm{~K}(1 \mathrm{eV})$. The trends seen at the lower temperatures in the $\left(\mathrm{C}^{+}, \mathrm{C}^{+}\right)$angles continue: The distribution broadens and the previously-well-defined peak at $\cos \alpha=$ 0.57 almost merges into the broad distribution. In contrast, the $\left(\mathrm{Br}^{+}, \mathrm{Br}^{+}\right)$pair distribution remains very similar to that at $400 \mathrm{~K}$. In the right-hand side of the figure, the maximum at $\cos \beta=0$ for the $\left(\mathrm{Br}^{+}, \mathrm{Br}^{+}, \mathrm{C}^{+}\right)$triplets has now completely disappeared. However, the distribution still does not drop to zero at $\cos \beta=0$. For the $\left(\mathrm{Br}^{+}, \mathrm{Br}^{+}, \mathrm{Br}^{+}\right)$triplets, the dissociation is still preferentially planar, with the distribution only slightly broadened (HWHM $22^{\circ}$ ) from the previous temperature.

When comparing the above results with the experiment, we noticed that the experimental $\cos \alpha$ distributions are dependent on the magnitudes of the momentum vectors. Therefore, a better comparison with the experiment can be obtained from Fig. 9, which shows how the angular distribution of the $\left(\mathrm{Br}^{+}, \mathrm{Br}^{+}\right)$pairs changes with the magnitude of the $\mathrm{Br}^{+}$ momenta, with the experiment in Fig. 9(a) and the simulation in Fig. 9(b). The latter was obtained by generating random charges between 0 and +2 for $\mathrm{Br}$ atoms and between 0 and +1 for $\mathrm{C}$ and $\mathrm{S}$ atoms. Also, $\mathrm{Br}$ atoms did not receive the maximum +2 charge before all atoms had became singly charged, and only the $\left(\mathrm{Br}^{+}, \mathrm{Br}^{+}\right)$pairs that carry charge +1 were used for the figure. The total charge range in the simulation was from +2 to +13 and the initial temperature $400 \mathrm{~K}$. The two-dimensional plot was constructed based on 50000 trajectories. For reference, horizontal dashed lines mark the $\mathrm{Br}^{+}$ion momenta from point charge explosion with 

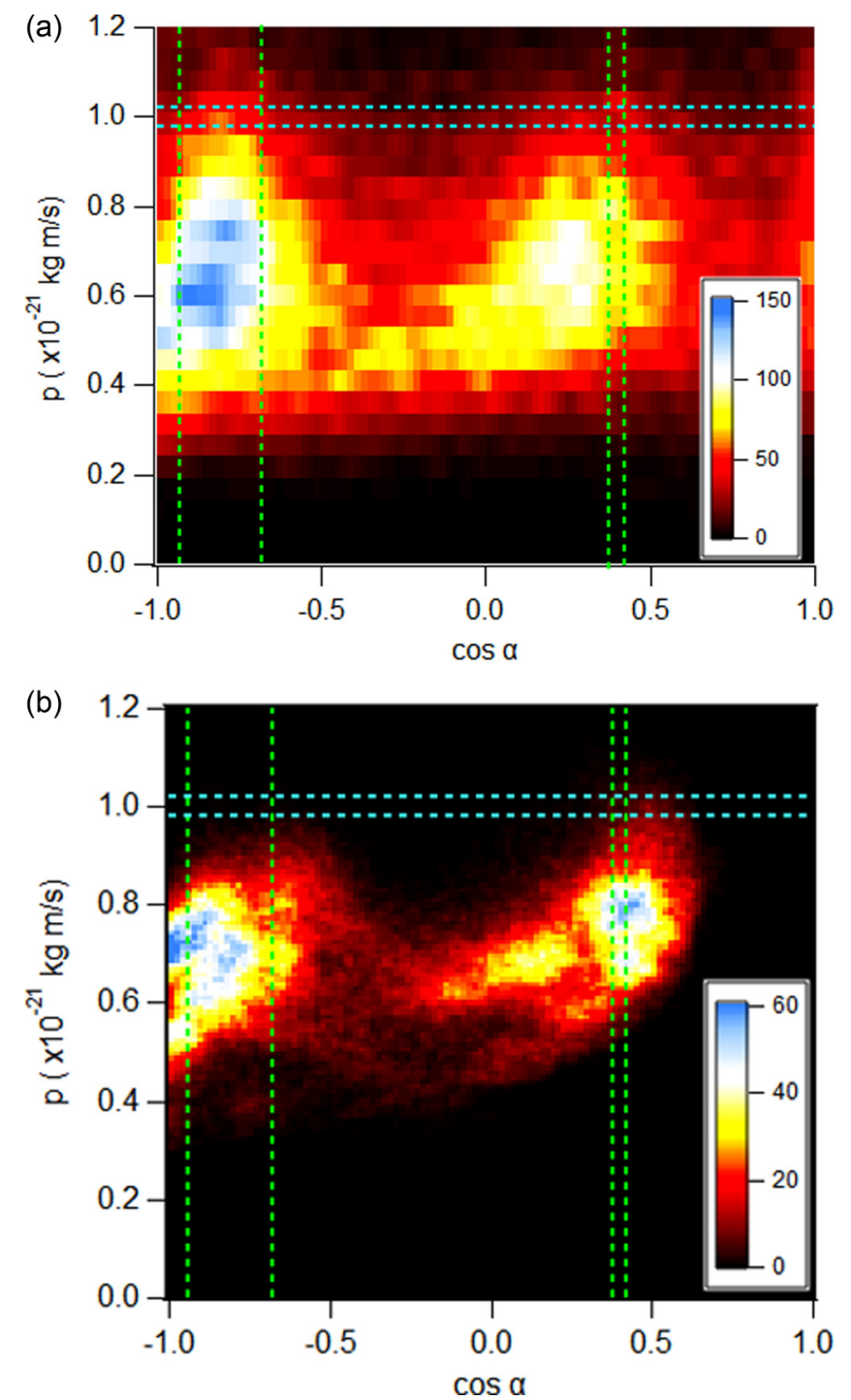

FIG. 9. (a) Experimental distribution of angles between two $\mathrm{Br}^{+}$momenta, dependent on the average momentum $\left[p\left(\mathrm{Br}_{1}{ }^{+}\right)+\right.$ $\left.p\left(\mathrm{Br}_{2}{ }^{+}\right)\right] / 2$ as the $y$ axis. The number of ion pairs in the distribution are color coded. Vertical lines denote the $\mathrm{C}-\mathrm{Br}$ bond angles in the parent molecule and horizontal lines mark the momentum values from simulation with $Q_{\text {tot }}=9$. (b) Simulated angular distributions.

$Q_{\text {tot }}=9$ and at $T=0 \mathrm{~K}$. Vertical dashed lines denote the bond angles form the neutral molecular geometry.

In general, considering the basic simplicity of the model and that even no charge dynamics was included and thus there were no adjustable empirical parameters except for the vibrational temperature, the overall agreement between the experiment and simulation is quite satisfactory. In both cases, it is seen that the distribution's maxima reflect better the bond angles in the neutral geometry as the total charge and the ion momenta increase. Thus, a faster and stronger Coulomb explosion of the $\mathrm{Br}^{+}$ions reflects the molecular geometry more closely. In the case of a weak explosion and low-momentum values of less than $5 \times 10^{-22} \mathrm{kgm} / \mathrm{s}$, both the experimental and simulated distributions shift towards fully anticorrelated vectors $(\cos \alpha<0)$. This is indeed expected from low total charge states, with $\cos \alpha$ approaching -1 for $Q_{\mathrm{tot}}=2$ at the lowest charge limit.

\section{Effects of charge dynamics}

On one hand, the ion trajectories and angular correlations in Coulomb implosion are highly sensitive to the initial vibrational motion of the atoms. On the other hand, the total kinetic energy release (KER), as well as the final momentum values in a typical Coulomb explosion as experienced by the $\mathrm{Br}^{+}$ions, is affected by the charge buildup and distribution timescales. Next we include these as empirical parameters in our model, in a manner slightly modified from the previous studies $[16,18]$.

The molecular charge builds up to the final total charge $Q_{\text {tot }}$ according to the equation

$$
Q(t)=Q_{\mathrm{tot}}\left[1-\exp \left(-\frac{t}{\tau}\right)\right],
$$

where $\tau$ is an empirical constant. In the present study, the main factor determining the charge buildup duration is the relatively long FEL pulse of estimated 70-fs duration. However, the charge buildup constant $\tau=70 \mathrm{fs}$ is likely to be too long, since strong absorption in the earlier part of the pulse increases both the molecular charge and the binding energy of the $\mathrm{Br} 3 d$ orbitals, lowering the absorption cross section as the photoelectron energy moves away from the giant resonance. Eventually, above $Q_{\text {tot }} \approx 10$ the ionization by $130-\mathrm{eV}$ photons from $\mathrm{Br} 3 d$ orbital becomes impossible altogether. We will use a tentative value of $\tau=50$ fs to explore the effects of charge buildup.

The initial charge, as $\mathrm{Br} 3 d$ core holes, will distribute to other atoms by Auger decay according to the equations

$$
\begin{aligned}
\frac{d Q_{\mathrm{Br}}}{d t} & =\frac{Q_{\mathrm{atom}}}{\tau} e^{-t / \tau}-R\left[Q_{\mathrm{Br}}(t)-Q_{\mathrm{C}, \mathrm{S}}(t)\right], \\
\frac{d Q_{\mathrm{C}, \mathrm{S}}}{d t} & =R\left[Q_{\mathrm{Br}}(t)-Q_{\mathrm{C}, \mathrm{S}}(t)\right],
\end{aligned}
$$

where $R$ is the charge transfer rate. In such dynamics, all $N$ atoms eventually obtain an equal charge $Q_{\text {atom }}=Q_{\text {tot }} / N$ and the charge dynamics is given in the integral form as

$$
\begin{aligned}
Q_{\mathrm{Br}}(t) & =\frac{Q_{\text {atom }}}{2 R \tau-1}\left[2(1-R \tau) e^{-t / \tau}-e^{-2 R \tau}\right]+Q_{\mathrm{atom}}, \\
Q_{\mathrm{C}, \mathrm{S}}(t) & =\frac{Q_{\mathrm{atom}}}{2 R \tau-1}\left(e^{-2 R \tau}-2 R \tau e^{-t / \tau}\right)+Q_{\mathrm{atom}} .
\end{aligned}
$$

In the present model, the atoms of the same type that have the same final charge also have equal fractional charges during the buildup. Thus, for $Q_{\text {tot }}=+9$, the charge buildup does not destroy the initial symmetry as all four carbon atoms and one sulfur have the same charge $Q_{\mathrm{C}, \mathrm{S}}(t)$ at any point of time and the four bromine atoms have equal $Q_{\mathrm{Br}}(t)$ charge.

Figure 10 presents momentum distributions from four charge dynamics scenarios: A, instantaneous charges; B, representing the present experiment; $\mathrm{C}$, same as B but with very fast charge transfer to carbon atoms; and D, very fast charge buildup and slow transfer. For the charge transfer rate in scenario B we used $R=1.5 \times 10^{14} \mathrm{~s}^{-1}$, derived from the $\mathrm{Br}$ $3 d$ core-hole lifetime of about 7 fs [27]. It is interesting to note that, apart from the expected reduction of the $\mathrm{Br}^{+}$ion 


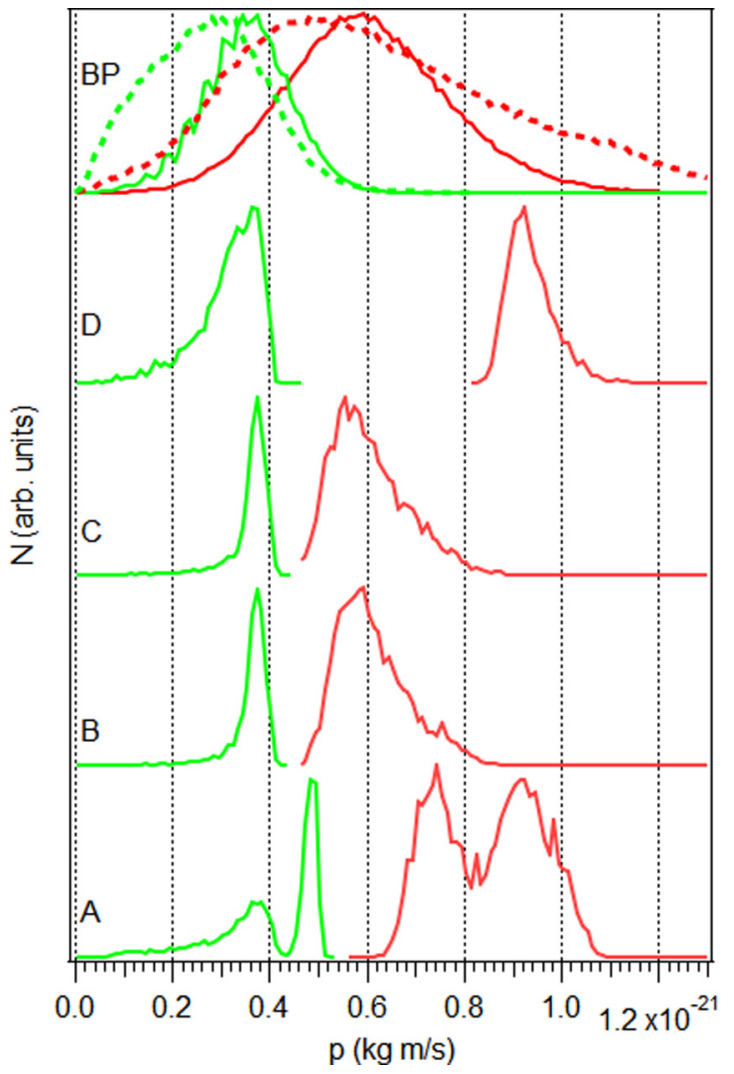

FIG. 10. Curves A-D are the simulated momentum distributions of $\mathrm{Br}^{+}$(red curves) and $\mathrm{C}^{+}$ions (green curves) with the total charge of $Q_{\mathrm{tot}}=9$ and $0.5 \mathrm{eV}$ of internal energy. In scenario A, $\tau=0$ fs and $R \rightarrow \infty$; in $\mathrm{B}, \tau=50$ fs and $R=1.5 \times 10^{14} \mathrm{~s}^{-1}$; in C, $\tau=50 \mathrm{fs}$ and $R \rightarrow \infty$; and in D, $\tau=1 \mathrm{fs}$ and $R=1 \times 10^{13} \mathrm{~s}^{-1}$. Here BP is a comparison of experiment (dotted curves) and simulation according to scenario $\mathrm{B}$, but with the total charge state given by a Poisson distribution with $\bar{Q}_{\text {tot }}=9$.

momenta when switching on charge dynamics (from A to B), the distributions also become less structured. Although the maxima for both the $\mathrm{C}^{+}$and $\mathrm{Br}^{+}$momentum distributions in simulation $\mathrm{B}$ are fairly close to the experimental maxima at $3 \times 10^{-22}$ and $5 \times 10^{-22} \mathrm{~kg} \mathrm{~m} / \mathrm{s}$, respectively (Fig. 3), this simulation at $Q_{\mathrm{tot}}=9$ is not for direct comparison (for which the whole range of total charges should be modeled). Scenario $\mathrm{C}$ demonstrates in turn that the charge flow from $\mathrm{Br}$ to other atoms is fast enough compared to the buildup time, so the charge equalization time does not need to be included. Scenario D shows a hypothetical situation where the slow transfer rate dominates the charge dynamics. In this case, the $\mathrm{Br}^{+}$ions that received near-instantaneous charge obtain high momenta, and carbon ions are more affected by the slow charge flow. In scenario D, the bromine atoms charge quickly to about +2 , before the charge slowly dissipates to other atoms, accounting for strong initial Coulomb forces and high $\mathrm{Br}^{+}$ion momenta.

Scenario B in Fig. 10 is the best approximation in the model for quantitative comparison with the experimental distributions (Fig. 3), yet it does not account for the variations of the total charge. The top curves BP in Fig. 10 provide such a comparison by assuming a Poisson distribution of $Q_{\text {tot }}$ with the average charge of +9 . Scenario B was simulated for all charges $Q_{\text {tot }}=+2$ to +17 and these distributions were added according to the weights from the Poisson distribution. One can see that after including the vibrational motion and charge dynamics, the modeled momentum distribution of $\mathrm{Br}^{+}$is well centered on the experimental one. Its width is primarily influenced by the choice of the $Q_{\text {tot }}$ distribution function, which may well be different from the assumed Poisson distribution. The agreement is worse between the experimental and simulated momenta of the $C^{+}$ion, with the former having a pronounced low-momentum region down to zero. In the case of Coulomb implosion, the low momentum is not necessarily correlated with low total charge (see Fig. 3). In simulations, near-zero momenta for $\mathrm{C}^{+}$are generated by the basic model for all total charges, with no vibrations and charge dynamics included (see Fig. 6(b)). Thus the discrepancy is not simply removed by adjusting the total charge distribution. As discussed in connection with Fig. 7, these are the carbon ions that undergo multiple in-plane scattering within the bromine cage, and this effect quickly vanishes with the addition of vibrations. A more detailed investigation (of angular correlations, for example) is hampered by the very high relative experimental error in $\frac{\Delta p}{p}$ of the near-zero-momentum ions. From the modeling side, one possible source of discrepancy is the handling of the internal energy as atomic velocities. In the Supplemental Material, we investigated other methods of distributing the energy over molecular vibrations [28]. While these more advanced treatments have minor effects on the resultant momentum and angular distributions, they offer no distinct improvements at the present level of detail in the available experimental data.

Finally, Fig. 11 shows the angular distributions obtained from scenario B in comparison with experiment. Constant background was subtracted from the experimental curve to account for the false coincidences. As for the ion momentum, the $\left(\mathrm{C}^{+}, \mathrm{C}^{+}\right)$and $\left(\mathrm{Br}^{+}, \mathrm{Br}^{+}\right)$pair angular distributions become more smeared out by slower charge dynamics, especially the sharp maximum at $\cos \alpha=-1$ seen in Fig. 7. There is good agreement of simulations with the experiment, except for one clear discrepancy: There is a high probability of codirectional $\mathrm{C}^{+}$ion emission $(\cos \alpha=1)$ in experiment, whereas in simulations this is suppressed by C-C Coulomb repulsion. In our statistical charge buildup model all $\mathrm{C}$ atoms charge concurrently, always maintaining equal charge. Once the $\mathrm{C}^{+}$ion leaves the molecular plane, the four Brions (which dissociate close to that plane) exert on it a concerted force close to the plane normal direction. However, the mutual repulsion of the $\mathrm{C}^{+}$ions leaving on the same side of the plane causes a widening of the angle $\alpha$ between their momentum vectors, thus suppressing the angular distribution at $\cos \alpha=1$. In experiment, photons are absorbed sequentially over the pulse duration and thus the $\mathrm{C}$ atoms become charged and leave the molecular plane at different times. Even though the four heavy $\mathrm{Br}$ atoms also become charged at different times during the pulse, due to their slow motion the timescale of the sequential charging does not destroy their original geometry and they still exert a concerted near-planenormal force on the $\mathrm{C}$ ions. In such a case, their mutual Coulomb repulsion of $\mathrm{C}^{+}$ions does not prevent codirectional emission. 

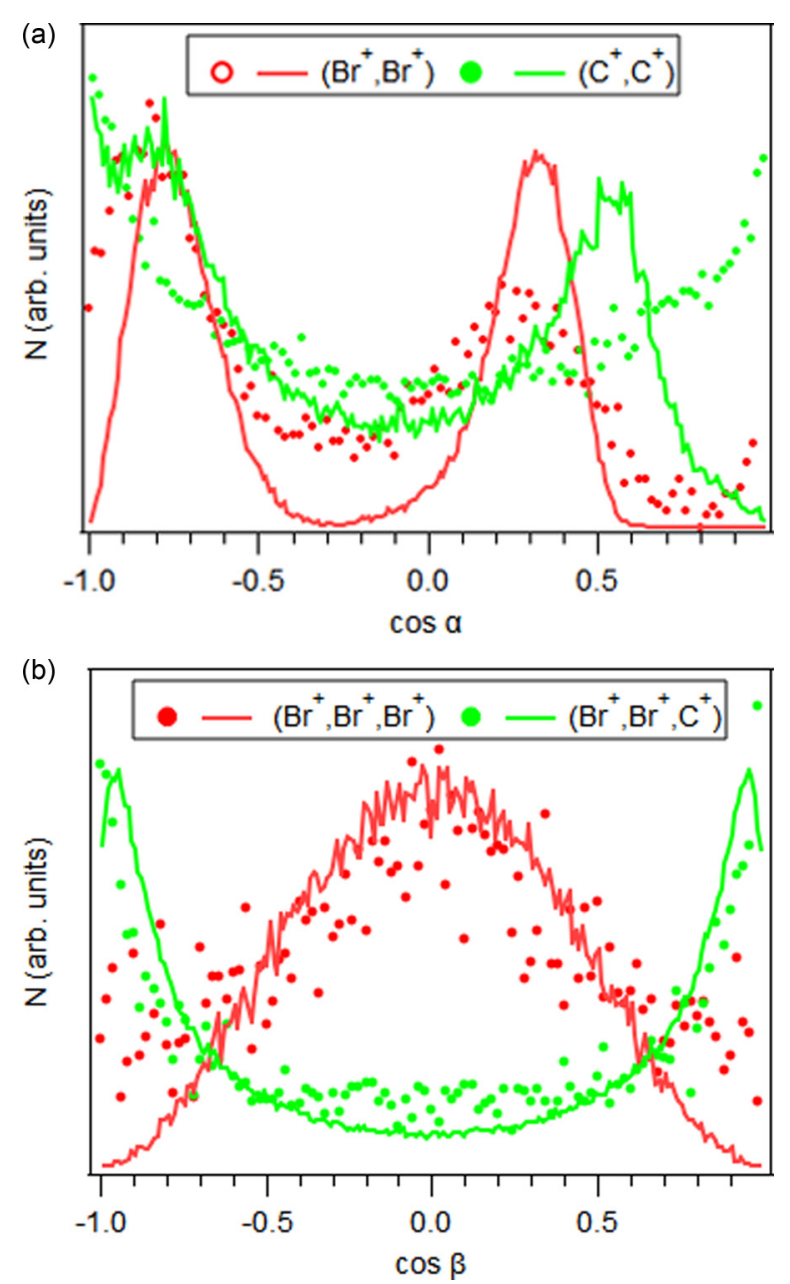

FIG. 11. Distributions of (a) $\cos \alpha$ for $\left(\mathrm{Br}^{+}, \mathrm{Br}^{+}\right)$and $\left(\mathrm{C}^{+}, \mathrm{C}^{+}\right)$ pairs and (b) $\cos \beta$ for $\left(\mathrm{Br}^{+}, \mathrm{Br}^{+}, \mathrm{Br}^{+}\right)$and $\left(\mathrm{Br}^{+}, \mathrm{Br}^{+}, \mathrm{C}^{+}\right)$triplets. Lines represent simulations as described in the text and markers are experimental distributions.

In comparing the planarity $(\cos \beta)$ of ion emission, the simulations of scenario $\mathrm{B}$ [Fig. 11(a)] agree very well with experiment for both $\left(\mathrm{Br}^{+}, \mathrm{Br}^{+}, \mathrm{C}^{+}\right)$and $\left(\mathrm{Br}^{+}, \mathrm{Br}^{+}, \mathrm{Br}^{+}\right)$ triplets.

\section{CONCLUSION}

Short and intense FEL pulses cause strong sequential multiphoton ionization of gas-phase tetrabromothiophene molecules, leading to high total charge states and extensive fragmentation. From three-dimensional ion momentum analysis, the momentum distributions and various two- and threeion angular correlations were determined for $\mathrm{Br}^{+}$and $\mathrm{C}^{+}$ions.

A comparison of the momentum distributions with a pointcharge dissociation model shows that charge states of up to $Q_{\text {tot }} \approx 13$ were reached. We chose $Q_{\text {tot }}=9$ with uniform charge distribution to all atoms for further simulations. This creates a very symmetric situation where the $\mathrm{C}^{+}$ions experience a Coulomb implosion with unique characteristics for ion momentum and angular distributions. This situation makes the dissociation trajectories of $\mathrm{C}^{+}$extremely sensitive to initial conditions such as thermal vibrational motion of atoms. By including initial thermal velocity in simulations, temperatures as low as $40 \mathrm{~K}$ completely alter the angular correlations of the departing fragments, with the $\mathrm{C}^{+}$ions popping out of the molecular plane and losing the relationship to the initial geometry. The momentum vector correlations, predicted by the model with instant charge creation but including vibrational motion, are in general in good agreement with the experiment, both clearly exhibiting the distinct properties of the Coulomb implosion of the thiophene ring vs the Coulomb explosion of the surrounding $\mathrm{Br}$ atoms.

Adding charge buildup time to the simulation has a significant effect on the total KER and the individual ion momenta, while the charge transfer time from the $\mathrm{Br} 3 d$ holes to the whole molecule has a minor impact under the present conditions. A close quantitative comparison of ion momentum distributions from the simulation and experiment is difficult because the total charge distribution is not well known, however the momenta in the Coulomb explosion of $\mathrm{Br}^{+}$appear to be in closer agreement than the momenta in the Coulomb implosion of $\mathrm{C}^{+}$. With further experimental improvements in low-momentum accuracy and with the reduction of residual ion background, the near-zero-momentum ions from Coulomb implosion in highly charged systems can be utilized for more advanced Coulomb implosion description including, e.g., molecular bonds and sequential dissociation processes. The influence of the added charge dynamics on the angular correlations is small, but still improves the quantitative agreement with the observations.

The 4BrThio molecule proved to be a sensitive test for modeling the fragmentation of highly charged molecular systems. Its bromine atoms exhibit typical geometry-determined Coulomb explosion, whereas the properties of the carbon ions allow studying the influence of the temperature. The detailed shapes of the momentum and angular distributions under Coulomb implosion conditions can potentially reveal more information on the interaction between nuclear and charge dynamics, for example, and could be used for further refinements of the theoretical model, but it would require a more detailed knowledge of the experimentally created total molecular charge distribution (or an ability to choose a particular total charge state) than was available for this study.

For future studies, it would be of interest to experimentally investigate the effects of the various temperatures (or molecular vibrations controlled by other means) on 4BrThio or other systems experiencing Coulomb implosion. Furthermore, specific vibrational excitations before or during the fragmentation, to which Coulomb explosions are typically insensitive, could be probed by Coulomb-implosion-type dynamics. Also, Coulomb implosions could be sensitive indicators of small initial geometry distortions of molecules in high-symmetry situations.

\section{ACKNOWLEDGMENTS}

This study was supported by the X-ray Free Electron Laser Utilization Research Project and the X-ray Free Electron Laser Priority Strategy Program of the Ministry of Education, Culture, Sports, Science and Technology of Japan (MEXT), by the Proposal Program of SACLA Experimental Instruments of RIKEN, by the research program "Dynamic 
alliance for open innovation bridging human, environment and materials" in Network Joint Research Center for Materials and Devices, by IMRAM project, and by the Japan Society for the Promotion of Science. E.K. and E.P. acknowledge financial support from the Academy of Finland and D.Y. acknowledges support from a Grant-in-Aid of Tohoku University Institute for Promoting Graduate Degree Programs,
Division for Interdisciplinary Advanced Research and Education. M.B. acknowledges financial support from the ASTRA project PER ASPERA Graduate School of Functional Materials and Technologies receiving funding from the European Regional Development Fund under a project at University of Tartu, Estonia. The authors also thank Dr. J. Niskanen for his help in improving the molecular-dynamics model.
[1] P. H. Bucksbaum, R. Coffee, and N. Berrah, The first atomic and molecular experiments at the Linac coherent light source x-ray free electron laser, Adv. At. Mol. Opt. Phys. 60, 239 (2011).

[2] J. Ullrich, A. Rudenko, and R. Moshammer, Free-electron lasers: New avenues in molecular physics and photochemistry, Annu. Rev. Phys. Chem. 63, 635 (2012).

[3] M. Yabashi, H. Tanaka, and T. Ishikawa, Overview of the SACLA facility, J. Synchrotron Radiat. 22, 477 (2015).

[4] C. Bostedt, S. Boutet, D. M. Fritz Z. Huang, H. J. Lee, H. T. Lemke, A. Robert, W. F. Schlotter, J. J. Turner, and G. J. Williams, Linac coherent light source: The first five years, Rev. Mod. Phys. 88, 015007 (2016).

[5] E. A. Seddon, J. A. Clarke, D. J. Dunning, C. Masciovecchio, C. J. Milne, F. Parmigiani, D. Rugg, J. C. H. Spence, N. R. Thompson, K. Ueda et al., Short-wavelength free-electron laser sources and science: A review, Rep. Prog. Phys. 80, 115901 (2017).

[6] M. N. Piancastelli, M. Simon, and K. Ueda, Present trends and future perspectives for atomic and molecular physics at the new $\mathrm{x}$-ray light sources, J. Electron Spectrosc. Relat. Phenom. 181, 98 (2010).

[7] B. Erk, R. Boll, S. Trippel, D. Anielski, L. Foucar, B. Rudek, S. W. Epp, R. Coffee, S. Carron, S. Schorb et al., Imaging charge transfer in iodomethane upon $\mathrm{X}$-ray photoabsorption, Science 345, 288 (2014).

[8] H. Fukuzawa, S.-K. Son, K. Motomura, S. Mondal, K. Nagaya, S. Wada, X.-J. Liu, R. Feifel, T. Tachibana, Y. Ito et al., Deep Inner-Shell Multiphoton Ionization by Intense X-Ray FreeElectron Laser Pulses, Phys. Rev. Lett. 110, 173005 (2013).

[9] L. Young, K. Ueda, M. Gühr, P. H. Bucksbaum, M. Simon, S. Mukamel, N. Rohringer, K. C. Prince, C. Masciovecchio, M. Meyer et al., Roadmap of ultrafast X-ray atomic and molecular physics, J. Phys. B 51, 32003 (2018).

[10] A. Rudenko, L. Inhester, K. Hanasaki, X. Li, S. J. Robatjazi, B. Erk, R. Boll, K. Toyota, Y. Hao, O. Vendrell et al., Femtosecond response of polyatomic molecules to ultra-intense hard X-rays, Nature (London) 546, 129 (2017).

[11] B. Erk, D. Rolles, L. Foucar, B. Rudek, S. W. Epp, M. Cryle, C. Bostedt, S. Schorb, J. Bozek, A. Rouzee et al., Ultrafast Charge Rearrangement and Nuclear Dynamics upon Inner-Shell Multiple Ionization of Small Polyatomic Molecules, Phys. Rev. Lett. 110, 053003 (2013).

[12] N. Berrah, Molecular dynamics induced by short and intense X-ray pulses from the LCLS, Phys. Scr. T169, 14001 (2016).

[13] B. F. Murphy, T. Osipov, Z. Jurek, L. Fang, S.-K. Son, M. Mucke, J. H. D. Eland, V. Zhaunerchyk, R. Feifel, L. Avaldi et al., Femtosecond x-ray-induced explosion of $\mathrm{C}_{60}$ at extreme intensity, Nat. Commun. 5, 4281 (2014).
[14] T. Tachibana, Z. Jurek, H. Fukuzawa, K. Motomura, K. Nagaya, S. Wada, P. Johnsson, M. Siano, S. Mondal, Y. Ito et al., Nanoplasma formation by high intensity hard X-rays, Sci. Rep. 5, 10977 (2015).

[15] B. Rudek, S.-K. Son, L. Foucar, S. W. Epp, B. Erk, R. Hartmann, M. Adolph, R. Andritschke, A. Aquila, N. Berrah et al., Ultra-efficient ionization of heavy atoms by intense $\mathrm{x}$-ray free-electron laser pulses, Nat. Photon. 6, 858 (2012).

[16] K. Motomura, E. Kukk, H. Fukuzawa, S-i Wada, K. Nagaya, S. Ohmura, S. Mondal, T. Tachibana, Y. Ito, R. Koga et al., Charge and nuclear dynamics induced by deep inner-shell multiphoton ionization of $\mathrm{CH}_{3} \mathrm{I}$ molecules by intense x-ray free-electron laser pulses, J. Phys. Chem. Lett. 6, 2944 (2015).

[17] T. Takanashi, K. Nakamura, E. Kukk, K. Motomura, H. Fukuzawa, K. Nagaya, S.-i. Wada, Y. Kumagai, D. Iablonskyi, Y. Ito et al., Ultrafast Coulomb explosion of the diiodomethane molecule induced by an x-ray free-electron laser pulse, Phys. Chem. Chem. Phys. 19, 19707 (2017).

[18] K. Nagaya, K. Motomura, E. Kukk, H. Fukuzawa, S. Wada, T. Tachibana, Y. Ito, S. Mondal, T. Sakai, K. Matsunami et al., Ultrafast Dynamics of a Nucleobase Analog Illuminated by a Short Intense X-Ray Free Electron Laser Pulse, Phys. Rev. X 6 , 021035 (2016).

[19] K. Nagaya, K. Motomura, E. Kukk, Y. Takahashi, K. Yamazaki, S. Ohmura, H. Fukuzawa, S. Wada, S. Mondal, T. Tachibana et al., Femtosecond charge and molecular dynamics of I-containing organic molecules induced by intense x-ray free-electron laser pulses, Faraday Discuss. 194, 537 (2016).

[20] E. Kukk, K. Motomura, H. Fukuzawa, K. Nagaya, and K. Ueda, Molecular dynamics of XFEL-induced photo-dissociation, revealed by ion-ion coincidence measurements, Appl. Sci. 7, 531 (2017).

[21] H. Fukuzawa, K. Nagaya, and K. Ueda, Advances in instrumentation for gas-phase spectroscopy and diffraction with shortwavelength free electron laser, Nucl. Instrum. Methods Phys. Res. Sect. A 907, 116 (2018).

[22] K. Motomura, L. Foucara, A. Czasch, N. Saito, O. Jagutzki, H. Schmidt-Böcking, R. Drner, X.-J. Liu, H. Fukuzawa, G. Prümper et al., Multi-coincidence ion detection system for EUV-FEL fragmentation experiments at SPring-8, Nucl. Instrum. Methods Phys. Res. Sect. A 606, 770 (2009).

[23] S. Owada, K. Togawa, T. Inagaki, T. Hara, T. Tanaka, Y. Joti, T. Koyama, K. Nakajima, H. Ohashi, Y. Senba et al., A soft x-ray free-electron laser beamline at SACLA: The light source, photon beamline and experimental station, J. Synchrotron Radiat. 25, 282 (2018).

[24] M. Yabashi, H. Tanaka, K. Tono, and T. Ishikawa, Status of the SACLA facility, Appl. Sci. 7, 604 (2017). 
[25] J. J. Yeh, Atomic Calculation of Photoionization Cross-Sections and Asymmetry Parameters (Gordon and Breach, Langhorne, 1993); J. J. Yeh and I. Lindau, At. Data Nucl. Data Tables 32, 1 (1985).

[26] T. Ebata, Study on the structure and vibrational dynamics of functional molecules and molecular clusters by double resonance vibrational spectroscopy, Bull. Chem. Soc. Jpn. 82, 127 (2009).
[27] T. Matila, R. Püttner, A. Kivimäki, H. Aksela, and S. Aksela, Auger decay widths of the ligand-field-split $\mathrm{Br} 3 \mathrm{~d}$ components in the $\mathrm{HBr}$ molecule, J. Phys. B 35, 4607 (2002).

[28] See Supplemental Material at http://link.aps.org/supplemental/ 10.1103/PhysRevA.99.023411 for a detailed description of the procedure for defining the initial conditions for molecular dynamics simulation. 